\title{
FORMACIÓN DEL DOCENTE Y SU ADAPTACIÓN AL MODELO TPACK
}

\section{TEACHER TRAINING AND TPACK MODEL ADAPTATION}

Francisco Lenin Morán Peña, Dr.

Universidad de Guayaquil, Guayaquil - Ecuador

Lenin.moranp@ug.edu.ec

\section{Francisco Enrique Morán Peña, Mtr.}

Universidad de Guayaquil, Ecuador

Francisco.moranp@ug.edu.ec

José Dionel Albán Sánchez, Mtr.

Universidad de Guayaquil, Ecuador

jose.albans@ug.edu.ec

\section{RESUMEN}

La carrera de Físico Matemático (FIMA) de la Facultad de Filosofía, Ciencias y Letras de la Educación de la Universidad de Guayaquil (FFUG), forma docentes para el ciclo de bachillerato en el área de la Física y las Matemáticas. El docente universitario que imparte clases en la Carrera de FIMA, debe tener diferentes tipos de competencias para dar a sus estudiantes alternativas de aprendizaje, contextualizado al mundo actual. La presente investigación tiene como objetivo, diagnosticar el tipo de educación que están recibiendo los estudiantes de la Carrera y si es posible la adaptación del modelo Technological, Pedagogical and Content Knowledge / Conocimiento Tecnológico, Pedagógico del Contenido (TPACK). Para lograr determinar si posible adaptar el modelo TPACK, se aplicó un modelo de cuestionario validado por docentes universitarios. Estos cuestionarios han sido aplicados a los estudiantes y docentes de la Carrera FIMA. Los resultados fueron tabulados y a continuación se aplicó el coeficiente de fiabilidad Alfa de Cronbach, aproximándose al valor máximo de uno, indicando la fiabilidad de los resultados. Además, se realizó varias entrevistas para contrastar estos resultados. De esta manera se da a conocer la presente investigación, poniendo a su consideración el proceso de la misma y sus conclusiones.

Palabras Claves: Formación Docente, Físico Matemático, Adaptación, TPACK.

\section{ABSTRACT}

The Physics Mathematical career/ Físico Matemático (FIMA) in the Faculty of Philosophy, Letters and Science Education at the University of Guayaquil/Facultad de Filosofía, Letras y Ciencias de la Educación de la Universidad de Guayaquil (FFUG), form teachers for High School cycle in the area of Physics and the Mathematics. The university teacher that teaches at the FIMA Career, should have different types of skills to give the students the learning alternative, contextualized the current world. The present investigation has as purpose; diagnose the type of education that FIMA students are receiving and if it is possible the adaptation of the model Technological, Pedagogical and Content Knowledge (TPACK). To achieve determine whether it is possible to adapt the model TPACK, applied model questionnaires validated by university teachers. These questionnaires have been applied to the students and teachers of the FIMA career. The results were tabulated and then the reliability coefficient alpha of Cronbach was applied, approaching the maximum value of one, indicating the reliability of the results. In addition, several interviews were conducted to compare these results. Thus it is disclosed this investigation, putting the process and the conclusions at your consideration.

Key Words: Teacher Training, Physics Mathematical, Adaptation, TPACK.

Recibido: 30/05/2017

Aceptado: 25/06/2017

Publicado: 30/06/2017 


\section{INTRODUCCIÓN}

El modelo TPACK (Technological, PedAgogical and Content Knowledge / Conocimiento Tecnológico, Pedagógico del Contenido) propuesto por Mishra P. y Koehler M.J., sobre este modelo indican:

Es el conocimiento sobre el Contenido de la materia, la Pedagogía que se debe aplicar y la tecnología más adecuada para la enseñanza. Sin embargo, en lugar de tratarlos como cuerpos de conocimiento separados, este modelo enfatiza adicionalmente la compleja interacción de estos tres cuerpos de conocimiento ${ }^{1}$.

EI TPACK, es un modelo en estudio y ha generado expectativa en el área de la formación docente, debido a la integración de los Conocimientos (Tecnológico, Pedagógico y Contenido). Además, los conocimientos que se crean con la integración de estos.

La Carrera de Físico Matemático (FIMA) de la Facultad de Filosofía, Ciencias y Letras de la Educación de la Universidad de Guayaquil (FFUG), forma docentes para el Bachillerato en las áreas de la Física y las Matemáticas. Se pretende conocer la adaptabilidad de la formación de los estudiantes al modelo TPACK. Para alcanzar este fin se plantea realizar un diagnóstico con cuestionarios dirigido a estudiantes y docentes de la Carrera. Luego se tabulará los resultados para proceder hacer un contraste con los informantes de la investigación. Al final se podrá observar las conclusiones que llegaron los investigadores.

\section{DESARROLLO}

\subsection{Formación Docente}

La formación docente para el bachillerato debe ser dialéctica, contextualizada a los cambios socio culturales dentro de entornos educativos pertinentes, para un aprendizaje integral.

El desarrollo profesional es un proceso permanente e integral de actualización psicopedagógica y en ciencias de la educación. Esto conduce al mejoramiento de sus conocimientos, habilidades y competencias ${ }^{2}$.

El docente debe tener en la actualidad competencias interculturales, tecnológicas, lingüísticas entre otras, que se deben poner en práctica. Para la Unesco ${ }^{3}$ "la formación profesional del docente será componente fundamental de esta mejora de la educación. No obstante, el desarrollo profesional del docente sólo tendrá impacto si se centra en cambios específicos del comportamiento de este en la clase y, en particular, si ese desarrollo es permanente y se armoniza con otros cambios en el sistema educativo".

En la formación docente, es primordial el conocimiento de las tecnologías educativas como parte global del desarrollo académico. Según Suárez, et al. ${ }^{4}$, "se relacionan con el conocimiento y habilidades en los diferentes recursos tecnológicos, mientras que las pedagógicas son aquellas que permiten al profesorado utilizar estos recursos tecnológicos en su diseño y desarrollo curricular".

Con una sociedad, que vive constantes cambios en su forma de existir en este mundo, las Tecnologías de la Información y Comunicación (TIC) han irrumpido en la educación. Para Rodera ${ }^{5}$ "La incorporación de metodologías de formación interactivas, que aprovechan el uso de las TIC, está empezando a exigir con firmeza la necesidad de establecer una innovación continua de los modelos formativos y educativos propuestos desde las universidades. Esta innovación se encuentra con la necesidad de establecer una innovación continua de los modelos formativos y educativos propuestos desde las universidades".

\subsection{Las TIC como medio de aprendizaje}

En la educación Superior, las TIC han realizado importantes aportaciones como medio de aprendizaje en:

- El proceso de planificación de las clases.

- La innovación en la didáctica.

- Herramientas para la presentación de contenidos.

- El acceso a recursos tecnológicos educativos (hardware y/o software).

- La interdisciplinaridad en el proceso de aprendizaje.

- Resultados en menos tiempos.

- La interactividad educativa.

- El desarrollo de habilidades.

- La motivación en la investigación.

Sobre las TIC Adell6 indica: 
Las TIC nos abren nuevas posibilidades. Múltiples herramientas para acceder a la información, gestionarla, reelaborarla, convertirla en conocimiento, y compartir artefactos culturales, $y$, sobre todo, para interactuar con otros docentes más experimentados, para participar en proyectos didácticos telecolaborativos, etc., nos brindan un nuevo "espacio" para el desarrollo profesional.

\subsection{La Pedagogía y la formación docente}

Se puede entender a la pedagogía como la forma de "transferir" la información para la formación de un individuo, para que este la transforme en conocimiento propio y se convierta en un ser humano productivo crítico, adaptado a un contexto definido con independencia de pensamiento.

\section{Alliaud y Estanislao ${ }^{7}$ entienden a la pedagogía} como:

Como la enseñanza, como el acto complejo de transmitir de manera intencional, metódica y sistemática fragmentos de mundo a las nuevas generaciones, y consideramos que es posible contribuir al diseño de una pedagogía de la formación docente, colocando a la enseñanza y al oficio en el centro de la reflexión.

Para cumplir con la idea de Alliaud y Estanislao, los docentes deben ser conocedores de modelos metodológicos y desarrollar competencias que ayuden a transmitir los contenidos programáticos del microcurrículo. Además, indican:

La pedagogía del oficio es también una socialización en competencias, capacidades y disciplinas, que parecen importantes para enseñar. Dadas las características de la práctica docente, tanto los aprendizajes más formalizados como los menos, sirven para aprender a enseñar. Saber enseñar es saber hacerlo y para poder hacerlo, como dijimos hay que ensayar, ejercitar y probar ${ }^{7}$.

\subsection{Contenidos del aprendizaje}

En la formación del docente de la carrera de FIMA se considera un $53,57 \%$ de las materias tienen una orientación a profundizar el contenido en el área de la Física y las Matemáticas.
Tabla 1. Clasificación de materias por eje de formación ${ }^{8}$

\begin{tabular}{|c|c|c|}
\hline $\begin{array}{c}\text { Eje de } \\
\text { Formación }\end{array}$ & $\begin{array}{c}\mathbf{N}^{\circ} \text { de } \\
\text { Materia }\end{array}$ & $\%$ \\
\hline Profesional & 30 & 53,57 \\
\hline Básicas & 17 & 30,35 \\
\hline Humanas & 9 & 16,07 \\
\hline Totales & 56 & 100,00 \\
\hline
\end{tabular}

Sobre los contenidos Escudero ${ }^{9}$ declara:

Los contenidos con los que se prepara al profesorado, los aprendizajes que logran con la formación, las oportunidades y condiciones con que cuentan para ir desarrollando sus concepciones y capacidades (metodologías, actividades, etc.), son tres componentes esenciales de cualquier plan de formación.

Es importante que el docente tenga dominio sobre los contenidos de la materia, porque profundiza sus conocimientos lo que facilitará paralelamente estudiar las metodologías más adecuadas para el aprendizaje en el aula de clases. "Los contenidos, no pueden estar ausentes en la formación del profesorado. Han de constituir la columna vertebral en las que insertar todos los contenidos, buscando el desarrollo de la comprensión y capacidades prácticas, así como esos valores y compromisos efectivos con los mismos" 9 .

\subsection{Modelo TPACK}

La aplicación de las TIC de manera descontextualizada, no reflejan el propósito de su creación (potencializar el proceso educativo), porque las TIC en sí, son medios tecnológicos y necesitan de una debida planificación metodológica para aplicarlas en clases.

En los últimos años, se ha conocido un modelo que une los Contenidos o la Disciplina, la Pedagogía o la Didáctica y la Tecnología usada como Tecnologías del Aprendizaje y del Conocimiento (TAC), en un enfoque integrador para mejorar el aprendizaje, al cual se le ha dado el nombre de Technological PedAgogical Content Knowledge (TPACK), o Conocimiento Tecnológico, Pedagógico del Contenido (CTPC).

Este modelo propuesto por Mishra P. y Koehler M.J., quienes en sus primeros estudios sobre el modelo indican:

Es el conocimiento sobre el Contenido de la materia, la Pedagogía que se debe aplicar y 
la tecnología más adecuada para la enseñanza. Sin embargo, en lugar de tratarlos como cuerpos de conocimiento separados, este modelo enfatiza adicionalmente la compleja interacción de estos tres cuerpos de conocimiento ${ }^{1}$

EI TPACK, es un modelo en estudio y ha generado expectativa en el área de la formación docente, debido a la integración de los Conocimientos (Tecnológico, Pedagógico y Contenido). Además, de los conocimientos que se crean con la integración de estos.

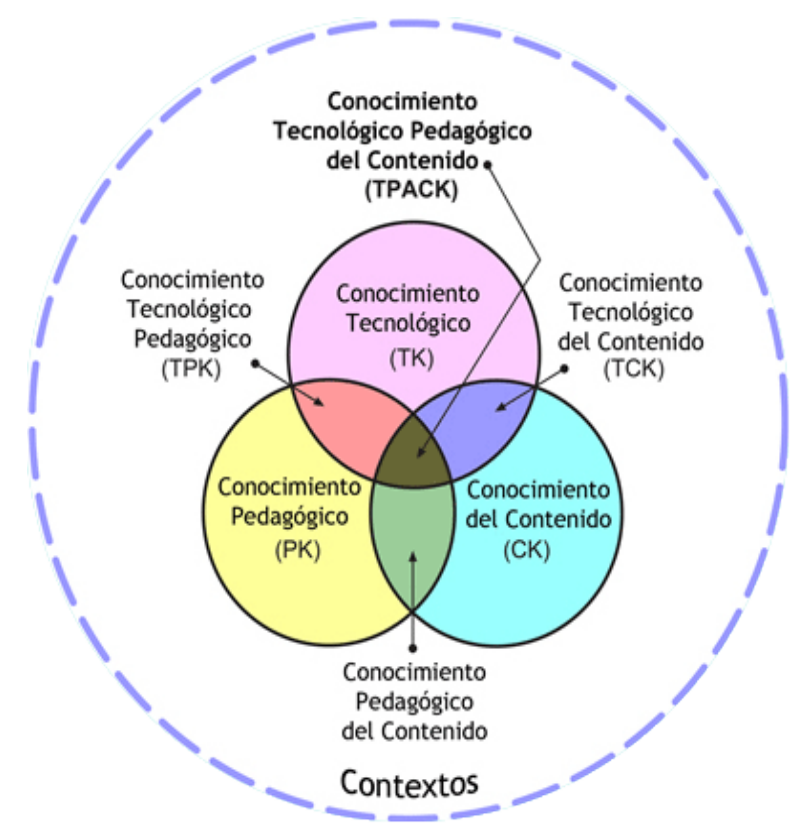

Figura 1. Modelo TPACK ${ }^{10}$

En esta figura se muestra el modelo TPACK, que identifica tres dimensiones del conocimiento básico de los profesores, que según Harris, Mishra y Koehler ${ }^{10}$ son Conocimiento del Contenido (CC), Conocimiento Pedagógico (CP) y Conocimiento Tecnológico (CT), pero el énfasis está puesto en las nuevas formas de conocimiento que se generan, a su vez, en la intersección entre cada uno de estos conocimientos CTC, CPC y CTP, para llegar a la intersección de las tres dimensiones que determina la situación óptima TPACK.

Es importante describir los conocimientos que integra el modelo TPACK, desde la primera versión de Mishra P. y Koehler M.J.

\section{Conocimiento del Contenido}

En la formación del docente, siempre es necesario que exista una selección adecuada de los contenidos académicos que van a fortalecer su dominio de temas relevantes que podrán en práctica en su profesión. "Los maestros deben conocer y comprender las materias que enseñan, incluyendo el conocimiento de hechos, conceptos, teorías y procedimientos centrales dentro de un campo dado; Conocimiento de marcos explicativos que organizan y conectan ideas"1.

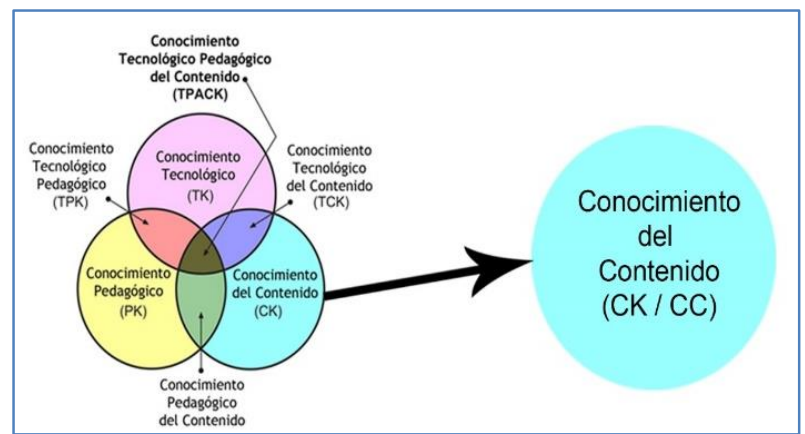

Figura 2. Conocimiento del Contenido

\section{Conocimiento Pedagógico}

El conocimiento Pedagógico (Didáctico), es el conocimiento que el docente en formación debe adquirir, para poder transmitir adecuadamente las enseñanzas a sus estudiantes, quienes deberán aprender didácticamente a construir sus propios conocimientos.

Es un conocimiento profundo de los procesos, prácticas o métodos de enseñanza aprendizaje y de cómo engloba, entre otras cosas, los propósitos, valores y objetivos educativos generales. Esta es una forma genérica de conocimiento que está involucrada en todos los temas de aprendizaje de los estudiantes, manejo del aula, desarrollo e implementación del plan de lección y evaluación del estudiante. Incluye conocimientos sobre técnicas o métodos que se utilizarán en el aula; La naturaleza del público objetivo1.

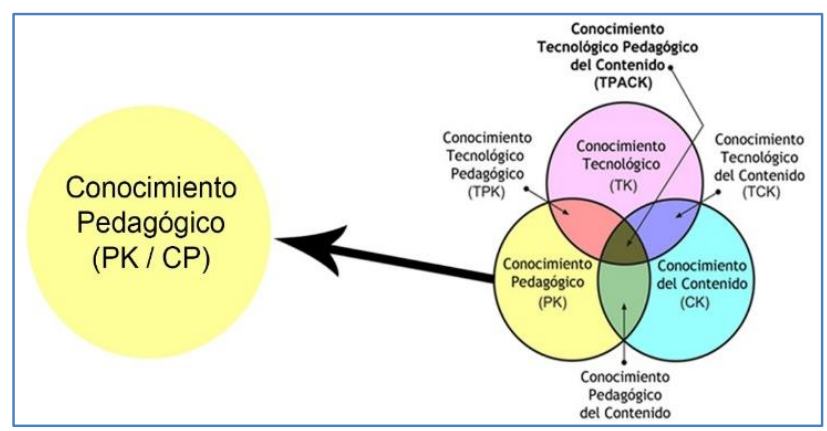

Figura 3. Conocimiento Pedagógico 


\section{Conocimiento Pedagógico del Contenido}

Esta intersección entre el Conocimiento Pedagógico y Conocimiento del Contenido, descrito por Mishra P. y Koehler M.J. es relacionado al de Shulman L.S. ${ }^{11}$ el conocimiento pedagógico es funcional a la enseñanza del contenido.

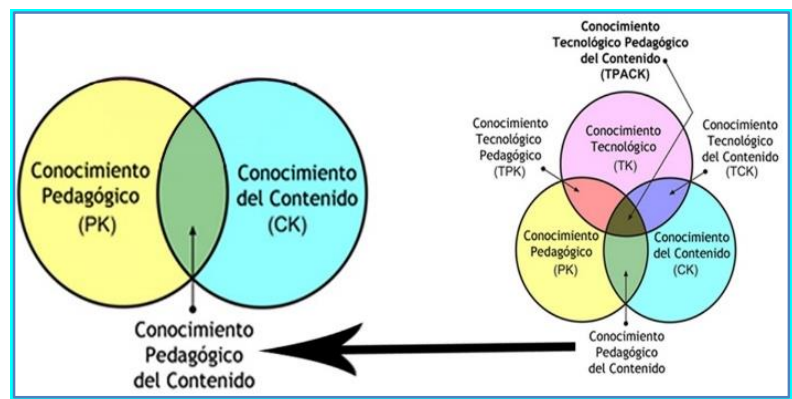

Figura 4. Conocimiento Pedagógico del Contenido

"EI PCK / CPC está interesado en la representación y formulación de conceptos, técnicas pedagógicas, conocimiento de lo que hace que los conceptos sean difíciles o fáciles de aprender, conocimiento del contenido previo de los alumnos y teorías de la epistemología"1.

\section{Conocimiento Tecnológico}

El conocimiento Tecnológico, implica tener habilidades para trabajar con tecnologías orientadas al área académica. Para Mishra P. y Koehler M.J.:

En el caso de las tecnologías digitales, esto incluye el conocimiento de los sistemas operativos y el hardware de la computadora y la capacidad de utilizar conjuntos estándar de herramientas de software como procesadores de texto, hojas de cálculo, navegadores y correo electrónico. TK / CT incluye conocimientos sobre cómo instalar y eliminar dispositivos periféricos, instalar y eliminar programas de software y crear y archivar documentos. La mayoría de los talleres de tecnología estándar y tutoriales tienden a centrarse en la adquisición de tales habilidades. Dado que la tecnología está cambiando continuamente, la naturaleza de los conocimientos tradicionales también debe cambiar con el tiempo ${ }^{1}$.

\section{Conocimiento Tecnológico del Contenido}

Cuando el Conocimiento Tecnológico se interseca con el Conocimiento del Contenido, relacionan la necesidad que el docente tiene con la tecnología para poder transmitir adecuadamente los temas de enseñanza en la clase. Por ejemplo, el programa GeoGebra, que es utilizado en los diferentes niveles educativos para la enseñanza de las matemáticas.

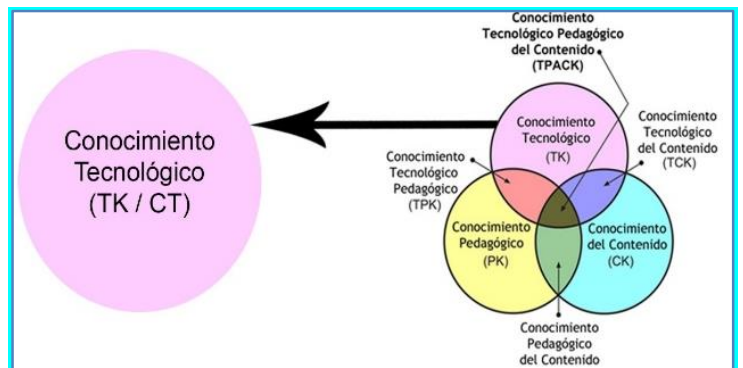

Figura 5. Conocimiento Tecnológico

En este sentido, el programa informático simplemente emula lo que se hizo antes al aprender geometría. Sin embargo, el programa de computadora hace más que eso. Al permitir a los estudiantes " jugar " con construcciones geométricas, también cambia la naturaleza de la geometría de aprendizaje; Las pruebas por construcción son una forma de representación en matemáticas que no estaba disponible antes de esta tecnología. Se pueden hacer argumentos similares para una gama de otros productos de software1.

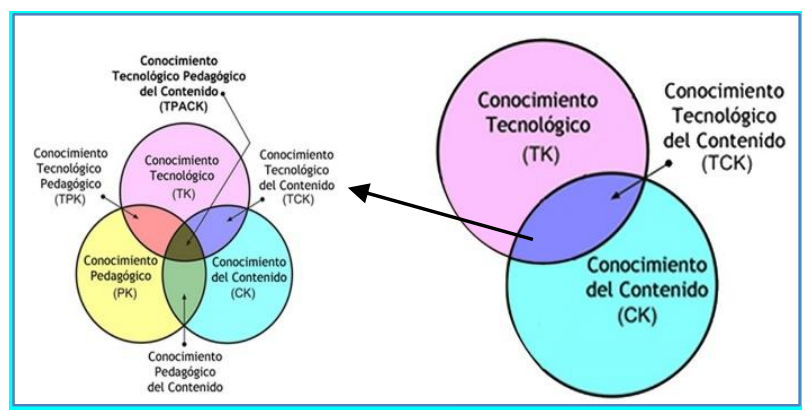

Figura 6. Conocimiento Tecnológico del Contenido

\section{Conocimiento Tecnológico Pedagógico}

Es importante para el docente poder saber utilizar didácticamente la tecnología, esta relación entre el conocimiento Tecnológico y el conocimiento Pedagógico, indica que se debe tener la capacidad para seleccionar adecuadamente las herramientas tecnológicas según la didáctica que se pretende aplicar en clase.

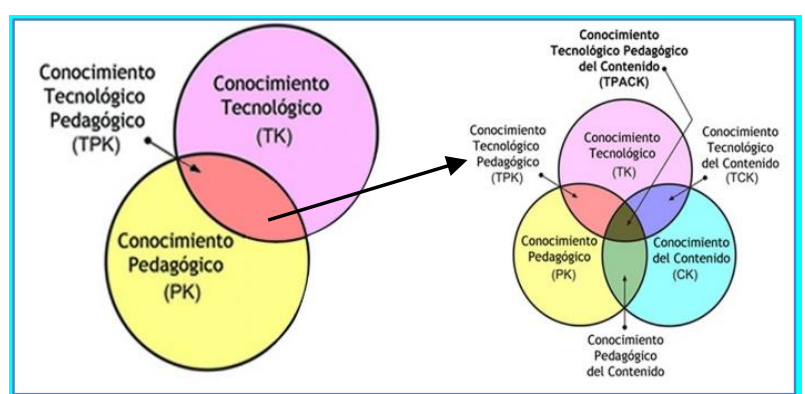

Figura 7. Conocimiento Tecnológico Pedagógico 


\subsubsection{TPACK y el aprendizaje}

En la formación de los docentes de física y matemáticas de la FFUG, es necesario que el aprendizaje tenga un contexto social y con sus influencias metodológicas contemporáneas, esto incluye los conocimientos que implica el TPACK, para Belfiori(12) "Teniendo en cuenta el modelo TPACK, los contenidos, problemas y asuntos de la Matemática son organizados, representados y adaptados para su enseñanza atendiendo a los intereses y habilidades de los alumnos, con lo cual, a veces, el contenido define la pedagogía y la tecnología que se utilizan y otras veces, la tecnología exige cambios en la pedagogía y habilita nuevas formas de representar un contenido. Incorporar tecnología no es lo mismo que sumar un nuevo contenido al programa, muchas veces cuestiona preceptos fundamentales de la disciplina - la pedagogía. Por lo que el modelo TPACK requiere de tres acciones fundamentales: primero, tener en claro cuáles son las necesidades pedagógicas, segundo, buscar y seleccionar recursos y, finalmente, definir el modo de uso de los recursos".

Los docentes de la Carrea FIMA, deben tener dominio profundo sobre los conocimientos tecnológicos, pedagógicos y de contenido (TPACK) para que la malla curricular que tiene más peso en las materias de Físico Matemático, pueda enlazar la didáctica y tecnología como ejes transversales de las materias, de tal forma que los estudiantes dentro de la clase de cualquier materia puedan tener todos los ámbitos del TPACK y formar una cultura de multiaprendizaje en el aula de clase.

Cabero(13) indica sobre los conocimientos que implica el TPACK "el docente debe tener formación y experiencia en diferentes aspectos de los tres componentes básicos del modelo, es decir, en lo referido al Conocimiento Tecnológico y de Contenidos, Conocimiento Pedagógico y de Contenido y Conocimiento Tecnológico Pedagógico".

\section{METODOLOGÍA}

Para la realización de esta investigación se utilizará una metodología Constructivista/Cualitativa, que consiste fundamentalmente en caracterizar un fenómeno o situación concreta indicando sus rasgos más peculiares o diferenciadores.
Para Latorre, Rincón y Arnal(14) "La metodología Constructivista/Cualitativa se orienta a describir e interpretar los fenómenos sociales, y por consiguientes los educativos, y se interesa por el estudio de los significados e intenciones de las acciones humanas desde la perspectiva de los propios agentes sociales. El enfoque de investigación de esta metodología se caracteriza por ser holístico, inductivo, ideográfico".

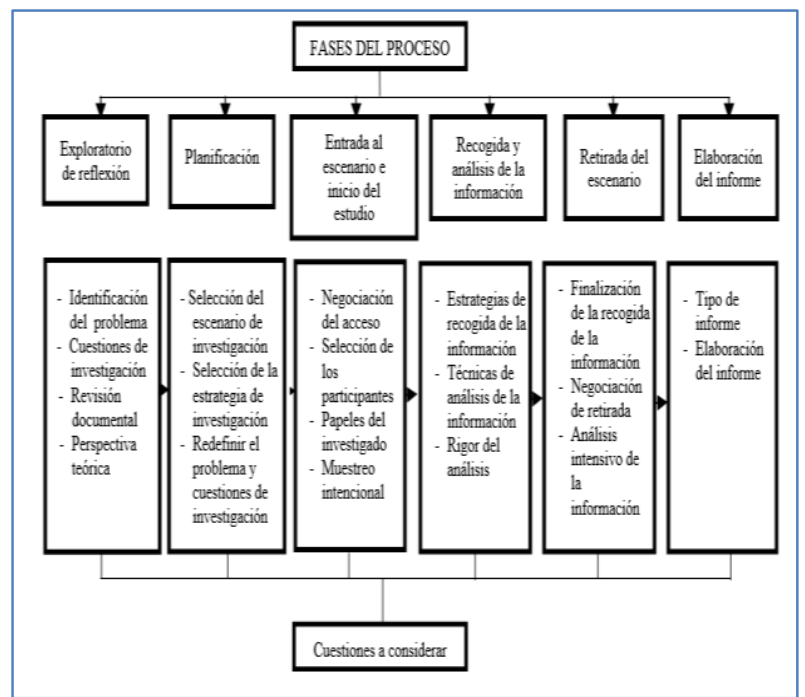

Figura 8. Proceso de investigación Constructivista / Cualitativa $^{14}$

El tipo de muestra será la no probabilística incidental, que selecciona de manera directa e intencional la muestra.

Tabla 2. Muestra poblacional

\begin{tabular}{|c|c|c|}
\hline Informante & Población & Muestra \\
\hline Docentes & 13 & 7 \\
\hline Estudiantes & 62 & 52 \\
\hline total & 75 & 59 \\
\hline
\end{tabular}

La selección de este tipo de muestra, es debido a que la población es reducida, y es factible aplicarla a todos los estudiantes y docentes. Para ser más precisos en la aplicación de los instrumentos, los autores revisaron las listas de estudiantes matriculados y su asistencia, de esta manera se pudo determinar que asisten 52 estudiantes a clases.

En el caso de los docentes, se debe mencionar que esta Carrera tiene dentro del sistema de estudio presencial dos formas de estudio: sistema anual y sistema semestral. Existen docentes en el sistema semestral, que en el momento de la aplicación de los instrumentos no estarán ejerciendo la cátedra por haber concluido su materia. 


\section{Técnicas}

Esta investigación constructivista/cualitativa aplicará las siguientes técnicas para la recolección de información: análisis de documentos, la observación, encuestas y entrevistas.

Indican Aravena, Kimelman, Micheli, Rodrigo, y Zúñiga ${ }^{15}$ :

Existen diversos tipos de entrevistas, pero todas se basan en la interacción social a través de la comunicación verbal. Las modalidades más utilizadas de entrevista cualitativa son: la entrevista individual (semiestructurada o en profundidad) y la entrevista grupal". Dentro del proceso de investigación serán entrevistados docentes y estudiantes.

Análisis de documentos: Para esta investigación se revisaron los documentos oficiales de la carrera de Físico Matemático:

1. Malla curricular

2. Datos académicos de los docentes

3. Reporte de la Autoevaluación de la Carrera

4. Listado de estudiantes matriculados

5. Listado de asistencia de los estudiantes

Método de Observación: Los autores estuvieron en las aulas de clases observando los procedimientos áulicos de los docentes. Entre lo que se observó tenemos:

1. Dominio del conocimiento de los contenidos de la materia

2. Dominio del conocimiento didáctico de los contenidos

3. Dominio del conocimiento tecnológico

\section{La integración de los conocimientos}

Además, se observó los recursos tecnológicos que se tiene para realizar las clases.

Entrevista: Se realizaron entrevistas de contraste de los resultados de las encuestas de los docentes y estudiantes

\section{RESULTADOS}

Se elaboraron cuestionarios dirigidos a los docentes y estudiantes de la Carrera de FIMA. Estas preguntas fueron seleccionadas para conocer como se está impartiendo las clases de las diferentes materias. En especial, lo referente a la aplicación de las Tecnologías Educativas.

Para realizar la encuesta se solicitaron permisos a las autoridades de la Facultad de Filosofía, Letras y Ciencias de la Educación de la Universidad de Guayaquil.

Una vez otorgado el permiso, se realizó una reunión con el Director de la Carrera de FIMA para que tenga conocimiento del procedimiento que se iba a realizar para aplicar los cuestionarios.

Se seleccionó de todos cursos de la Carrera de FIMA a 52 estudiantes, se les aplicó la encuesta, con las preguntas (P) que a continuación mencionamos:

\section{El docente en el aula de clases:}

P.1.- Es claro con los contenidos de la materia.

P.2.- Aplica técnicas didácticas para impartir clases.

P.3- Utiliza recursos tecnológicos en clase.

P.4.- Utiliza la tecnología más adecuada para el aprendizaje de la materia.

\begin{tabular}{|c|c|c|c|c|c|c|c|c|c|c|c|c|}
\hline \multirow{2}{*}{ Escala / Pregunta } & \multicolumn{2}{|c|}{ P.1 } & \multicolumn{2}{|c|}{$P .2$} & \multicolumn{2}{|c|}{ P.3 } & \multicolumn{2}{|c|}{$P .4$} & \multicolumn{2}{|c|}{$\boldsymbol{P . 5}$} & \multicolumn{2}{|c|}{ PROMEDIO } \\
\hline & $f$ & $\%$ & $f$ & $\%$ & $f$ & $\%$ & $f$ & $\%$ & $f$ & $\%$ & $f$ & $\%$ \\
\hline luy en Desacuerdo & 4 & 7,7 & 3 & 5,8 & 1 & 1,9 & 1 & 1,9 & 3 & 5,8 & 2,4 & 4,62 \\
\hline En desacuerdo & 10 & 19,2 & 6 & 11,5 & 7 & 13,5 & 7 & 13,5 & 4 & 7,7 & 6,8 & 13,08 \\
\hline Indiferente & 3 & 5,8 & 7 & 13,5 & 7 & 13,5 & 12 & 23,1 & 10 & 19,2 & 7,8 & 15,02 \\
\hline De acuerdo & 27 & 51,9 & 24 & 46,2 & 25 & 48,1 & 21 & 40,4 & 25 & 48,1 & 24,4 & 46,94 \\
\hline Muy de acuerdo & 8 & 15,4 & 12 & 23,1 & 12 & 23,1 & 11 & 21,2 & 10 & 19,2 & 10,6 & 20,4 \\
\hline Total & 52 & 100 & 52 & 100 & 52 & 100 & 52 & 100 & 52 & 100 & 52 & 100 \\
\hline
\end{tabular}

Tabla 3. Resultados de la encuesta dirigida a los estudiantes 
Tabla 4. Estadísticas de fiabilidad

\section{Alfa de Cronbach $\quad$ N. de elementos}

,835 5

P.5.- Guía y ayuda en el uso de tecnologías y aprendizajes de contenidos.

\section{Análisis de los resultados (estudiantes)}

Después de realizar la encuesta a los estudiantes, se procedió a tabular los datos. Con los resultados de las encuestas, los investigadores regresaron a las aulas de la Carrera para hacer un contraste de los resultados. De donde los autores pueden analizar lo siguiente:

P.1.La sumatoria de los porcentajes de las escalas Muy de acuerdo y De acuerdo es de $66,98 \%$. Los estudiantes indican que los docentes son claros con los contenidos de sus materias, que si aplican técnicas didácticas para impartir sus clases. Los autores en su proceso de observación de documentos de la Carrera de Físico Matemático, detalló en la Tabla 1, que existe un $53,57 \%$ de materias que se orientan al fortalecimiento de los contenidos de las materias del área de la Física y las Matemáticas. Esto explica que los estudiantes cuando ejercen su profesión de docente, tengan un amplio dominio sobre estos, y sus estudiantes pueden reconocerlo en esta pregunta. El docente de esta Carrera, para su ingreso a la Facultad, debe ganar un concurso de méritos. Este concurso, pondera con mayor puntaje los conocimientos de contenidos de las materias afines a la Física y las Matemáticas; por este motivo, esta pregunta tiene un alto porcentaje a favor de los docentes de la Carrera.

P.2.La sumatoria de los porcentajes de las escalas Muy de acuerdo y De acuerdo es del $69,3 \%$. Este valor es superior al de la P.1., los autores pudieron revisar los currículos de los docentes de la Carrera y observó que la formación de todos está relacionada a las Ciencias de la Educación, esto explica el alto porcentaje de aceptación de los procesos didácticos del docente.
P.3.El $71,2 \%$ de los estudiantes, contestó estar De acuerdo o Muy de acuerdo en la utilización de recursos de tecnológicos en clases, los autores pudieron contrastar este resultado en una entrevista con los docentes. Los docentes indicaron que debido a que su formación estaba más basada en los contenidos de las materias y las didácticas para su aplicación, ellos tuvieron que realizar de manera particular cursos de actualización en el área de las tecnologías educativas. Sobre las tecnologías indicaron, que es una debilidad en el proceso de formación del docente y que deberían integrarse de mejor manera dentro de la malla curricular.

P.4. El $61.6 \%$ de los estudiantes están Muy de acuerdo o De acuerdo en la adecuada aplicación de las tecnologías en el proceso de aprendizaje de la materia. Los docentes al tener una formación basada en los contenidos de las materias y las didácticas para su aplicación, han podido integrar esos conocimientos a los conocimientos tecnológicos aprendidos de manera particular. Esto ha permitido, que el docente pueda seleccionar las herramientas tecnológicas adecuadas a los contenidos y a la didáctica a utilizar para el aprendizaje de cada temática.

P.5. El $67,3 \%$ de los estudiantes están Muy de acuerdo o De acuerdo, en que el docente guía y ayuda en el uso de las tecnologías y el aprendizaje de contenidos. Es importante, que el docente logré enseñar de manera transversal la aplicación de las tecnologías educativas, esto ayuda suplir el bajo porcentaje de materias que tienen en la malla curricular en el área de las tecnologías.

El promedio del porcentaje de la escala Indiferente es de $15,2 \%$. En el contraste realizado con los estudiantes, sentirse indiferentes a estas temáticas, porque piensan que esto no va a cambiar y que es algo normal dentro de la educación.

Por otra parte, la sumatoria de los promedios de los porcentajes de las escalas Muy en desacuerdo y En desacuerdo es de 17,70\%. En el contraste realizado con los estudiantes, dijeron que los docentes no estaban aplicando bien las tecnologías dentro del aula de clases y algunos de los profesores no las usan. 
Reconocen que es necesario la aplicación de las tecnologías en el aula de clases.

Para conocer la fiabilidad de los resultados, se aplicó el coeficiente de fiabilidad Alfa de Cronbach, que indica que mientras más cerca del valor 1 es más fiable el resultado, como en este caso es de 0,835 , es fiable. Además, que se considera que los valores superiores a 0,7 son suficientes para reconocer la fiabilidad del resultado.

\section{Encuesta a docentes}

Se seleccionó a 7 docentes de 13 que tiene la Carrera, para realizar la encuesta, con las siguientes preguntas:

P.1. Sé usar en mis materiales docentes para el aula, estrategias que combinan contenidos, tecnologías y enfoques docentes sobre los cuales he aprendido.

P.2. Puedo guiar y ayudar a otras personas a coordinar el uso de contenidos, tecnologías y enfoques docentes en mi centro docente.

P.3. Puedo seleccionar tecnologías que mejoran el contenido de las lecciones.

\section{Análisis de los resultados (docentes)}

Después de realizar las encuestas y su respectiva tabulación, se procedió hacer un contraste de los resultados con los docentes:

Tabla 5. Resultados de la encuesta dirigida a los Docentes

\begin{tabular}{|c|c|c|c|c|c|c|c|c|}
\hline \multirow{2}{*}{ Escala / Pregunta } & \multicolumn{2}{|c|}{$P .1$} & \multicolumn{2}{|c|}{ P.2 } & \multicolumn{2}{|c|}{ P.3 } & \multicolumn{2}{|c|}{ PROMEDIO } \\
\hline & $f$ & $\%$ & $f$ & $\%$ & $f$ & $\%$ & $f$ & $\%$ \\
\hline Muy en Desacuerdo & 2 & 28,5 & 2 & 28,6 & 2 & 28,5 & 2 & 28,6 \\
\hline En desacuerdo & 0 & 0 & 0 & 0 & 0 & 0 & 0 & 0 \\
\hline Indiferente & 0 & 0 & 0 & 0 & 0 & 0 & 0 & 0 \\
\hline De acuerdo & 2 & 28,6 & 4 & 57,1 & 2 & 28,6 & 2,67 & 38,1 \\
\hline Muy de acuerdo & 3 & 42,9 & 1 & 14,3 & 3 & 42,9 & 2,33 & 33,3 \\
\hline Total & 7 & 100 & 7 & 100 & 7 & 100 & 7 & 100 \\
\hline
\end{tabular}

Tabla 6. Estadísticas de fiabilidad

\section{Alfa de Cronbach $\quad$ N. de elementos}

P.1. La sumatoria de los porcentajes de las escalas Muy de acuerdo y De acuerdo es de $71,5 \%$. Como se indicó en el análisis de las preguntas de los estudiantes, se realizó un contraste a docentes y estudiantes para conocer más sobre las respuestas realizadas a estas encuestas. Los docentes tienen una formación basada en el dominio de contenidos y la didáctica para aplicarlos, pero en el caso de la tecnología, ellos han tenido que de manera particular actualizarse, esto ha dado como resultado que los docentes puedan integrar estos conocimientos para el uso adecuado en las clases.

P.2. El $71,4 \%$ de los docentes indican que pueden guiar y ayudar a otras personas a coordinar el uso de sus conocimientos. Es una parte importante de la identidad del docente, que debido a su formación en Ciencias de la Educación puede integrar sus conocimientos para ayudar en su institución, en las preguntas realizadas a los estudiantes alcanzó un $67,3 \%$ la ayuda que el docente les brinda en la clase.

P.3. Los docentes en un $71,5 \%$ saben seleccionar las tecnologías para sus clases. En estos casos el dominio de las tecnologías, es importante para que puedan mejorar el aprendizaje de contenido. Los docentes han aprendido que es necesario saber aplicar de manera adecuada sus conocimientos con la integración de las tecnologías educativas, que 
como se indicó antes, han sido aprendidas de manera particular.

El promedio del porcentaje de la escala Muy en desacuerdo es de $28,6 \%$. Los docentes indican que su formación no estaba dirigida a la aplicación de las tecnologías.

\section{CONCLUSIONES}

La formación docente, es importante para una educación de calidad; los procesos académicos que se realizan para alcanzar este fin, deben estar cuidadosamente seleccionados. El estudio del modelo TPACK, para su posible aplicación en la Carrera de Físico Matemáticos, traería una nueva opción para los docentes, en busca de un enfoque que permita desarrollar los aprendizajes de sus estudiantes en el aula de clases. Una vez realizado este estudio, los autores pueden concluir que:

Es lamentable, que en la actualidad los docentes todavía deban formarse en el área de la Tecnologías Educativas (TE) con recursos propios, debido a que, en sus procesos de formación, fueron ignoradas las TE.

Los docentes sobre el problema de fatal de TE dentro de formación, se actualizan sobre las nuevas tecnologías, la mayoría lo hace de manera particular.

Existe una actitud positiva en los docentes para resolver sus problemas tecnológicos.

El modelo TPACK, si es posible adaptarlo a esta Carrera, debido que los docentes tienen los conocimientos necesarios en los contenidos de las materias, la didáctica para impartir esos contenidos y saben aplicar las tecnologías adecuadas para mejorar el aprendizaje. Es necesario que se capacite a los docentes en la aplicación del modelo.

\section{REFERENCIAS}

1. Mishra P, Koehler MJ. Technological pedagogical content knowledge: A framework for teacher knowledge. Teach Coll Rec. 2006;108(6):1017-54.

2. Nacional A. Ley Orgánica de Educación Intercultural Internet]. Ecuador; 2011 p. 46. Available from: http://educacion.gob.ec/wpcontent/uploads/downloads/2012/08/LOEl.pdf

3. Unesco. Normas UNESCO sobre Competencias en TIC para Docentes
[Internet]. Http://Www.Oei.Es. 2008. Available from:

http://www.oei.es/tic/UNESCOEstandaresDoc entes.pdfSuárez J, Almerich G, Gargallo B, Francisco Aliaga. Las competencias del profesorado en TIC: estructura básica. Educ XX1. 2013;16:39-62.

4. Rodera A. Profesores 2.0 en la universidad del siglo XXI. Universitat Oberta de Catalunya (UOC); 2012.

5. Adell J, Castañeda L. El desarrollo profesional de los docentes en entornos personales de aprendizaje (PLE). La práctica Educ en la Soc la Inf Innovación a través la Investig [Internet]. 2011;83-95. Available from:

http://digitum.um.es/xmlui/bitstream/10201/24 647/1/CastanedaAdell2011 preprint.pdf

6. Alliaud A, Estanislao A. Iniciarse en la docencia. Los gajes del oficio de enseñar. Profesorado Rev Currículum y Form Profr. 2009;13(1):89-100.

7. Reporte Autoevaluación Carrera F. Facultad de Filosofía, Universidad e Guayaquil [Internet]. Guayaquil; 2015. Available from: http://www.filosofia.edu.ec/

8. Escudero J. La formacion del profesorado de Educacion Secundaria: contenidos y aprendizajes docentes. Rev Educ. 2009;350:79-103.

9. Harris J, Mishra P, Koehler M. Teachers' Technological Pedagogical Content Knowledge and Learning Activity Types: Curriculum-Based Technology Integration Reframed. J Res Technol Educ [Internet]. 2008;41(4):393-416. Available from: http://eric.ed.gov/?q=technology+teacher+ed ucators\&ff $1=$ subTechnology+Integration\&id= EJ844273

10.Shulman LS. Shulman (1987).pdf. Vol. 57, Harvard Educational Review. 1987. p. 1-21.

11.Belfiori L. Uso del modelo TPACK por alumnos de un profesorado de matemática. Acta Latinoaméricana Matemática Educ. 2015;28:1733-40.

12. Cabero J. La Formación del profesorado en TIC: modelo TPACK. Publidisa. Tecnologías S de RA y N, editor. Sevilla; 2014. 161 p.

13. Latorre A, Rincon D, Arnal J. Bases metodológicas de la investigación educativa [Internet]. 2nd ed. Experencia E, editor. Barcelona; 2005. 135 p. Available from: http://www.edicionesexperiencia.com/fitxa.ph p?id_fitxa $=51$ Aravena M, Kimelman E, Micheli $B$, Rodrigo $T$, Zúñiga J. Investigación educativa I. 1st ed. AFEFCE- Universidad de ARCIS, editor. Santiago de Chile; 2006. 1-415 p. 